\author{
United Nations Educational Scientific and Cultural Organization \\ and \\ International Atomic Energy Agency
}

THE ABDUS SALAM INTERNATIONAL CENTRE FOR THEORETICAL PHYSICS

\title{
RELATIVISTIC BEAMING AND ORIENTATION EFFECTS IN CORE-DOMINATED QUASARS
}

\author{
A.A. Ubachukwu ${ }^{1}$ \\ Dept. of Physics and Astronomy, University of Nigeria, Nsukka, Nigeria \\ and \\ The Abdus Salam International Centre for Theoretical Physics, Trieste, Italy \\ and \\ A. E. Chukwude \\ Dept. of Physics and Astronomy, University of Nigeria, Nsukka, Nigeria.
}

\begin{abstract}
In this paper, we investigate the relativistic beaming effects in a well-defined sample of coredominated quasars using the correlation between the relative prominence of the core with respect to the extended emission (defined as the ratio of the core- to the lobe- flux density measured in the rest frame of the source) and the projected linear size as an indicator of relativistic beaming and source orientation. Based on the orientation-dependent relativistic beaming and unification paradigm for high luminosity sources in which the Fanaroff-Riley class-II radio galaxies form the unbeamed parent population of both the lobe- and core-dominated quasars which are expected to lie at successively smaller angles to the line of sight, we find that the flows in the cores of these core-dominated quasars are highly relativistic, with optimum bulk Lorentz factor, $\gamma_{\text {opt }} \sim 6-16$, and also highly anisotropic, with an average viewing angle, $\sim 9^{0}-16^{0}$. Furthermore, the largest boosting occurs within a critical cone angle of $\approx 4^{0}-10^{0}$. The results suggest that relativistic bulk flow appears to extend to kilo-parsec scales in these sources.
\end{abstract}

MIRAMARE - TRIESTE

July 2002

\footnotetext{
${ }^{1}$ Regular Associate of the Abdus Salam ICTP.
} 


\section{Introduction}

The phenomenology of active galactic nuclei $(A G N s)$ involves a supermassive black hole which releases relativistic outflows of energetic particles by accretion of matter through an accretion disk surrounded by an optically thick torus. These relativistic outflows form well-collimated symmetric twin jets or beams that feed the radio lobes (Rees 1971; Blandford and Rees 1974; Scheuer 1974; Blandford and Königl 1979). The interaction of the head of the beams/jets with the intergalactic medium produces the observed synchrotron lobe emission (Scheuer 1977). The classification of $A G N s$ depends on the power and geometry of the central engine as well as the jet/disk orientation with respect to our line of sight.(e.g. Antonucci 1993; Gopal-Krishna 1995; Falcke et al. 1995a,b; Urry and Padovani 1995). Thus, many properties of quasars and AGNs can be attributed to relativistic Doppler and geometric projection effects at small angles to our line of sight.

In low frequency surveys, the emission from high luminosity extragalactic radio sources is dominated by the lobe which usually have steep spectra (spectral index, $\alpha \geq 0.5, S_{\nu} \sim \nu^{-\alpha}$ ). These sources include the lobe-dominated quasars (LDQs) and Fanaroff and Riley (1974) classII (FRII) galaxies. The lobe emission is usually assumed to be isotropic so that radio source samples selected on the basis of their lobe emission should be orientation-unbiased. This means that, for the lobe- dominated sources, the ratio of the core flux density to that of the lobe (R) should not exceed unity (i.e. $R \leq 1$ ).

In contrast, high frequency source samples appear to be dominated by their core emissions so that radio sources selected from high frequency surveys tend to contain mostly core-dominated sources characterized by flat spectra $(\alpha<0.5)$ due to synchrotron self-absorption. These high luminosity radio sources are largely quasars and are called core-dominated quasars (CDQs). For these sources, the core emission depends on the viewing angle and can be Doppler boosted if the source axis is oriented close to the line of sight (i.e. $R>1$ ). In addition, the projected linear sizes $(D)$ of the $C D Q s$ are expected to be foreshortened due to geometrical projection effects at small viewing angles. The notable exception to the core-dominated objects observable at high frequencies are the compact steep spectrum sources which are of galactic dimensions and whose core emission appears to be orientation-invariant (Fanti et al. 1990).

Several statistical tests have been carried out which confirmed that $R$ is indeed a good statistical measure of relativistic beaming in the cores of high luminosity sources (e.g. Orr and Browne 1982; Kapahi and Saikia 1982; Hough and Readhead 1989; Saikia and Kulkarni 1994; Saikia et al. 1995). In previous papers (Ubachukwu 1998; 2002), we studied the statistical consequences of relativistic beaming and geometric projection effects in high luminosity lobedominated sources using their observed $R-D$ data. In this follow-up paper, we wish to extend the $R-D$ analyses to their core-dominated counterparts. 


\section{Doppler Boosting and Geometrical Projection Effects in Core- Dominated Sources}

The simplest relativistic beaming and radio source unification model predicts that both the projected linear size, $D$, and the core dominance parameter, $R$, should depend on the viewing angle according to the following equations;

$$
D=D_{0} \sin \phi
$$

and

$$
R=f \gamma^{-n}\left[(1-\beta \cos \phi)^{(-n+\alpha)}+(1+\beta \cos \phi)^{(-n+\alpha)}\right],
$$

where $D_{0}$ is the intrinsic size of the source, $f$ is the ratio of the intrinsic core luminosity to the unbeamed extended luminosity, $\beta$ is the velocity of the radiating material in units of the velocity of light, $\alpha$ is the spectral index and $\gamma=\left(1-\beta^{2}\right)^{-1 / 2}$ is the bulk Lorentz factor. The exponent $n$ depends on whether the radiating material consists of a continuous jet $(n=2)$ or blobs $(n=3)$; in general $2 \leq n \leq 3$ if the emission is isotropic in the rest frame of the source. For the purposes of the present study which involves the core-dominated sources, we shall assume $\alpha=0$ throughout. At small angles to the line of sight, relativistic beaming in active galactic nuclei $(A G N s)$ is fundamentally characterized by the Doppler factor (the ratio of the observed core luminosity to it's emitted value) given by,

$$
\delta=[\gamma(1-\beta \cos \phi)]^{-1} .
$$

Generally, the relativistic beaming hypothesis is based on two parameters, the bulk Lorentz factor/jet velocity $(\gamma / \beta)$ and the viewing angle, $\phi$. Equation (2) therefore suggests that the distribution of $R$ should provide us with an indication of the range of values of viewing angle as well as the Lorentz factor which can used to test specific beaming models once $f$ is known. Consequently, the mean angle to the line of sight can be calculated from the mean value $\left(R_{m}\right)$ of the R-distribution (from equation (2)) through

$$
\cos \phi_{m} \approx 1-\left(\frac{2^{n-1} R_{m}}{R_{T}}\right)^{-1 / 2}
$$

(for $\gamma>>1$ ), where $R_{T}=\frac{2 f}{\gamma^{n}}$ is the value of $R$ at $\phi=90^{\circ}$ (i.e. the value of $R$ for a source whose radio axis lies along the plane of the sky). The last equation is actually to a first approximation and is applicable to beamed sources whose radio axes are expected to lie close to the line of sight.

In addition, equation(3) has the implication that, for angles between $\phi=0^{\circ}$ and some critical angle, $\phi_{c r i t}$, the relativistic boosting is optimized and this can be obtained by setting $\frac{d \delta}{d \beta}=0$. This yields (e.g. Vermeulen and Cohen 1994; Ubachukwu 1999)

$$
\phi_{c r i t} \approx \sin ^{-1}\left(\frac{1}{\gamma_{o p t}}\right)
$$


where $\gamma_{o p t}$ is the Lorentz factor which can be derived from equation(2) as

$$
\gamma_{o p t} \approx\left[\frac{1}{2^{n-1}}\left(\frac{R_{\max }}{R_{T}}\right)\right]^{1 / 2 n}
$$

Here, $R_{\max }=R\left(\phi=0^{\circ}\right) \approx f(2 \gamma)^{n}$.

Furthermore, comparing equation(1) with equation(2) shows that $R$ should be anti-correlated with $D$. A graph of $R$ against $D$ for a well-defined source sample is expected to yield,

$$
R=R_{\max }-m D
$$

The last two equations suggest that we can deduce the value of the beaming parameter, $\gamma$, from the regression analyses of $R$ on $D$ for any assumed model once $R_{T}$ is known for a given source sample. This ofcourse presupposes that the observed $R-D$ correlation is entirely due to relativistic beaming. However, Linear sizes of extragalactic radio sources have been known to undergo cosmological evolution (e.g. Barthel and Miley 1988; Kapahi 1989; Neeser et al. 1995), but whether the observed evolution is real or an artifact of the luminosity selection effects often present in most bright source samples is still unclear (see Singal 1993; Nilsson et al. 1993; Ubachukwu and Ogwo 1998). Nevertheless, this effect if present, should be accounted for before $D$ could be used to test the relativistic beaming and radio source orientation scenarios. Following Ubachukwu (2002), we test these expectations for a sample of core-dominated sources in the following section.

\section{Analyses and Results}

The present analyses were based on a well-defined sample of powerful core-dominated sources compiled by Murphy et al. (1993). All of the sources have $5 \mathrm{GHz}$ core flux densities, $S>1 J y$. This sample consists of both BL Lacs and quasars which are variable sources. The quasar subsample, which is of interest for the present work, comprises 54 sources with complete $R$ and $D$ information.

The median value data for the $R$-distribution for the subsample is $R_{m}=12.6$. Orr and Browne (1982) have shown that the $R$-distribution for radio loud quasars is consistent with $R_{T}=0.024$, i.e. if the $L D Q s$ form the parent population of the $C D Q s$. This would imply (from equation(4)), $\phi_{m} \approx 14^{\circ}$ for $n=2$ or $\phi_{m} \approx 23^{\circ}$ for $n=3$. However, as noted by Padovani and Urry (1992), and Urry and Padovani (1995), there appear to be too few $L D Q s$ to form the parent population of the $C D Q s$. In fact Barthel $(1989,1994)$ has used the relative number densities and size distributions of radio galaxies and quasars in the $3 C R$ sample of Laing et al. (1983) to argue that high luminosity radio galaxies and quasars are the same objects seen from different orientation angles. We shall therefore adopt $R_{T}=0.003$ which appears to be consistent with the FRII-LDQ-CDQ unification scheme for high frequency surveys (see Padovani and Urry 1992; Simpson 1996; Morganti et al. 1997; Ubachukwu 2002). Using $R_{T}=0.003$ together with $R_{m}=12.6$ in equation (4) gives $\phi_{m} \approx 9^{\circ}$ for $n=2$ or $\approx 16^{o}$ for $n=3$. 
To check for possible evolutionary effects, we show the $R-z$ and $D-z$ plots in Fig. 1 and Fig. 2 respectively (where $z$ is the redshift). The two plots show no discernible trend. Linear regression analyses give correlation coefficient, $r \sim 0.2$, for each case implying a lack of any significant redshift dependence. Removal of the outlier, $1803+784$, weakens the correlation further (though not significantly) so we can hence conclude that evolution, if present, is very negligible and proceed to use the $R-D$ correlation to test the beaming hypotheses.

Fig. 3 shows the $R-D$ plot for the present sample (without the outlier). Although the plot shows no obvious general trend, the upper envelope $R-D$ function (which shows the locus of the maximum core dominance parameter as a function of the projected linear size) is welldefined. This function is usually attributed to relativistic beaming and geometric projection effects at small angles with respect to the line of sight (see Ubachukwu 1998; 2002). Linear regression analysis of the upper envelope $R$-data against $D$ in four ranges of $D: D<50 \mathrm{kpc}$; $50 \leq D \leq 100 k p c ; 100<D \leq 150 k p c$ and $D>150 k p c$, gives $R_{\max }=375.64$ with $r \sim 0.9$. Using $R_{\max }=375.64$ and $R_{T}=0.003$ in equation(6) gives the bulk Lorentz factor (for optimum boosting), $\gamma_{o p t} \approx 16$ and $\sim 6$ for $n=2$ and 3 respectively. The corresponding critical beaming angle from equation $(5)$ becomes, $\phi_{\text {crit }} \approx 4^{o}$ (for $n=2$ ) or $\approx 10^{\circ}$ (for $n=3$ ).

\section{Discussion}

Two main results can be derived from the analyses presented in the preceding section. These two results were based on the simple assumption that the relativistic beam emanating from $A G N s$ is narrow and can be described with a single Lorentz factor, $\gamma$, once a flow model, which can either be in the form of a continuous jet $(n=2)$ or in form of individual blobs of plasma $(n=3)$, is adopted. Each of these models leads to different values of $\gamma$ and viewing angle, $\phi$. A more general situation may therefore be obtained in-between these two flow models $(2 \leq n \leq 3)$ and this will form the basis for our discussion. It could be noted however, that smaller values of $n$ imply higher $\gamma$-values but lower values of the viewing angle, $\phi$. Furthermore, it was assumed that the Fanaroff-Riley class-II radio galaxies form the unbeamed parent population of both the $L D Q s$ and $C D Q s$, with the later being the most beamed counterpart (cf Barthel 1989). However, analysis based on the Orr and Browne (1982) model in which the LDQs form the parent population of $C D Q s$ leads to a lower value of the Lorentz factor but a higher value of the orientation angle.

The first main result comes from the distribution of the core dominance parameter, $R$ which shows that, on the average, $C D Q s$ are inclined at $\phi \approx 9^{\circ}-16^{\circ}$, with respect to the line of sight. The second result is the presence of a strong anti-correlation between $R$ and the apparent linear size, $D$ for the upper envelope $R-D$ data. This correlation was shown to be consistent with an optimum Lorentz factor, $\gamma_{o p t} \approx 6-16$, with corresponding critical beaming angle (i.e. the largest angle for optimum boosting), $\phi_{\text {crit }} \approx 4^{\circ}-10^{\circ}$. These results are all consistent with the 
orientation-dependent relativistic beaming and radio source unification paradigm in which the flows from the cores of $C D Q s$ are expected to be highly relativistic (even at large scales) and in which Doppler effects generate anisotropic radiation patterns at close angles to our line of sight. The results are also in quantitative agreement with previous works as discussed below.

Based on the assumption that the $L D Q s$ form the unbeamed parent population of radio loud quasars, Orr and Browne (1982) showed that the radio luminosity function of $C D Q s$ is consistent with a core Lorentz factor, $\gamma=5$ (see also Kapahi and Saikia 1982; Hough and Readhead 1989). Similar result $(\gamma=4)$ was also obtained by Ubachukwu (1999) based on the core dominance - proper motion data for $L D Q s$ as the parent population of $C D Q s$ (see Vermeulen 1995). However, as mentioned in the preceding section, there appears to be too few $L D Q s$ to form the parent population of the CDQs; the relativistic beaming and radio source unification hypotheses imply that the parent population must be large. It is therefore more appropriate to derive the beaming and orientation parameters of $C D Q s$ based on the FRII radio galaxies as the parent population and not $L D Q s$.

Padovani and Urry (1992), and Urry and Padovani (1995) have argued that if the high luminosity radio galaxies form the parent population of both the $L D Q s$ and $C D Q s$ then, it is not possible to fit the luminosity function with a single Lorentz factor but a distribution with a weighted mean, $\gamma_{m} \sim 11$. They showed that the $C D Q s$ should have their radio axes within $\sim 14^{o}$ (with an average $\sim 9^{\circ}$ ) of the line of sight. They also noted that high values of the Lorentz factor and lower values of $n$ are necessary to provide better fit to the observed luminosity function. On the other hand, Ghisellini et al. (1993) used the synchrotron self-Compton model to estimate the value of $\gamma$ from the observed and predicted x-ray flux densities and found $\gamma \approx 10$ and a viewing angle of $\sim 8^{\circ}$ for $C D Q s$. Using the correlation between the accretion disk (UV) luminosity and the radio core emission of a sample of radio-loud quasars, Falcke (1995a) obtained a distribution of $3 \leq \gamma \leq 10$. These results are in close agreement with those obtained here.

\section{Conclusion}

We have investigated a simple statistical consequence of the relativistic beaming model in which, due to Doppler boosting and geometric projection effects at small viewing angles in core-dominated sources, the core dominance parameter, $R$, is expected to correlate inversely with the projected linear size, $D$, if the former is to be used as an orientation indicator. Our result shows a strong anti-correlation in the $R-D$ upper envelope data which offers both qualitative and quantitative support to the hypotheses that jets in core-dominated quasars are both highly relativistic as well as anisotropic. This is consitent with the orientation-dependent relativistic beaming and unification paradigm for high luminosity radio sources in which the $F R I I$ radio galaxies form the parent population of the lobe- and core-dominated quasars, with the latter being the most beamed counterpart. 


\section{Acknowledgements}

This work was done while AAU was visiting the Abdus Salam International Centre for Theoretical Physics as a Regular Associate. He is grateful to the Swedish International Development Agency (SIDA) for a generous contribution towards the visit. 


\section{References}

Antonucci, R. 1993, ARA\&A, 31, 473

Barthel, P. D. 1989, ApJ, 336, 606

Barthel, P. D. 1994, in Bicknell G. V., Dopita, M. A., Quinn, P. eds, The First Stromlo Symposium: Physics of Active Galaxies. Cambridge Univ. Press, Cambridge, p.175

Barthel, P. D. and Miley, G. K. 1988, Nat., 333, 318

Blandford, R. D. and Königl, A. 1979, ApJ., 232, 24

Blandford, R. D. and Rees, M. J. 1974, MNRAS, 169, 395

Falcke, H., Malkam, M. A. and Biermann, P. L. 1995a, A\&A, 298, 375

Falcke, H., Gopal-Krishna and Biermann, P. L. 1995b, A\&A, 298, 395

Fanaroff, B. L., and Riley, J. M. 1974, MNRAS, 167, 13p

Fanti, R., Fanti, C., Schilizzi, R. T., Spencer, R. E., Nan Rendong, Parma, P., van Breugel, W.

J. M. and Venturi, T. 1990, A\&A, 231, 333

Ghisellini, G., Padovani, P., Celotti, A. and Maraschi, L. 1993, ApJ, 407, 67

Gopal-Krishna 1995, Proc. Natl. Acad. Sci. 92, 11399

Hough, D. H. and Readhead, A. C. S. 1989, AJ, 98, 1208

Kapahi, V. K. and Saikia, D. J. 1982, JAA, 3, 465

Laing, R. A., Riley, J. M. and Longair, M. S. 1983, MNRAS, 204, 151

Morganty, R., Oosterloo, T. A., Reynolds, J. E., Tadhunter, C. N. and Migenes, V. 1997, MNRAS, 284, 541

Murphy, D. W., Browne, I. W. A. and Perley, R. A. 1993, MNRAS, 264, 298

Neeser, M. J., Eales, S. A., Law-Green, J., Leahy, J. P. and Rawlings, S. 1995 ApJ, 451, 76

Nilsson, K., Valtonen, M. J., Jaakola, T. 1993, ApJ, 413, 453

Orr, M. J. L. and Browne, I. W. A. 1982, MNRAS, 200, 1067

Padovani, P. and Urry, C. M. 1992, ApJ, 387, 449

Rees, M. J. 1971, Nat. 2229, 312

Saikia, D. J., Jeyakumar, S., Wiita, P. J., Sanghera, H. and Spencer, R. E. 1995, MNRAS. 276, 1215

Saikia, D. J. and Kulkani, V. K. 1994, MNRAS, 270, 897

Scheuer, P. A. G. 1974, MNRAS, 166, 513

Scheuer, P. A. G. 1977, in Radio Astronomy and Cosmology, IAU Symp 74, eds D. L. Jauncy (Reidel, Dordrecht), p. 434

Simpson, C. 1996, Vistas Astron., 40, 57

Singal, A. K. 1993, MNRAS, 263, 139

Ubachukwu, A. A. 1998, Ap\&SS, 257, 23

Ubachukwu, A. A. 1999, Publ. Astron. Soc. Aust, 16, 130

Ubachukwu, A. A. 2002, Ap\&SS, 279, 251

Ubachukwu, A, A. and Ogwo, J. N. 1998, AJP, 51, 143 
Urry, C. M. and Padovani, P. 1995, PASP, 107, 803

Vermeulen, R. C. 1995, in Quasars and AGN: High Resolution Imaging, ed M. H. Cohen and

K. I. Kellermann (Washington DC: National Academy of Science), p. 11385

Vermeulen, R. C. and Cohen, M. H. 1994, ApJ, 430, 467 


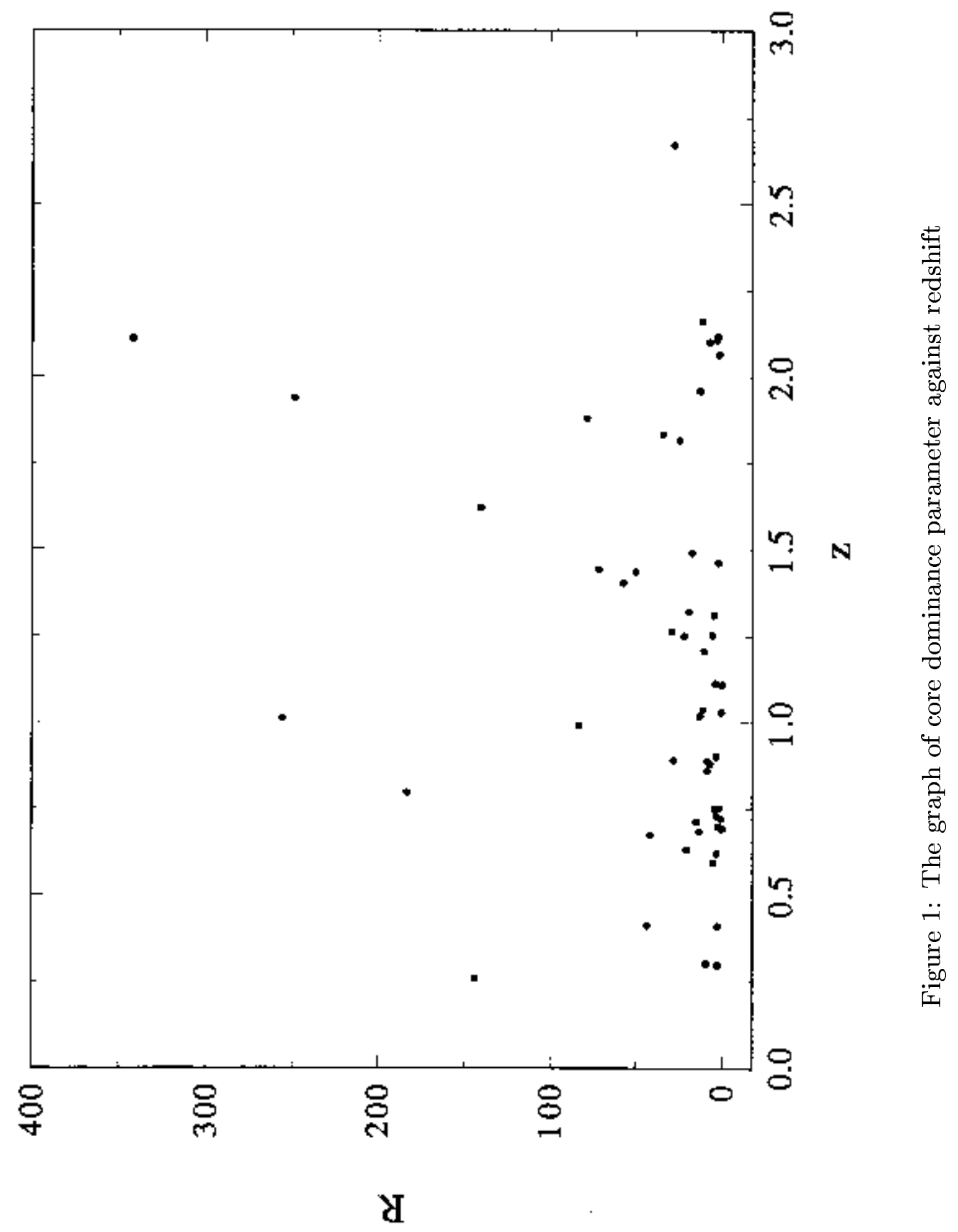




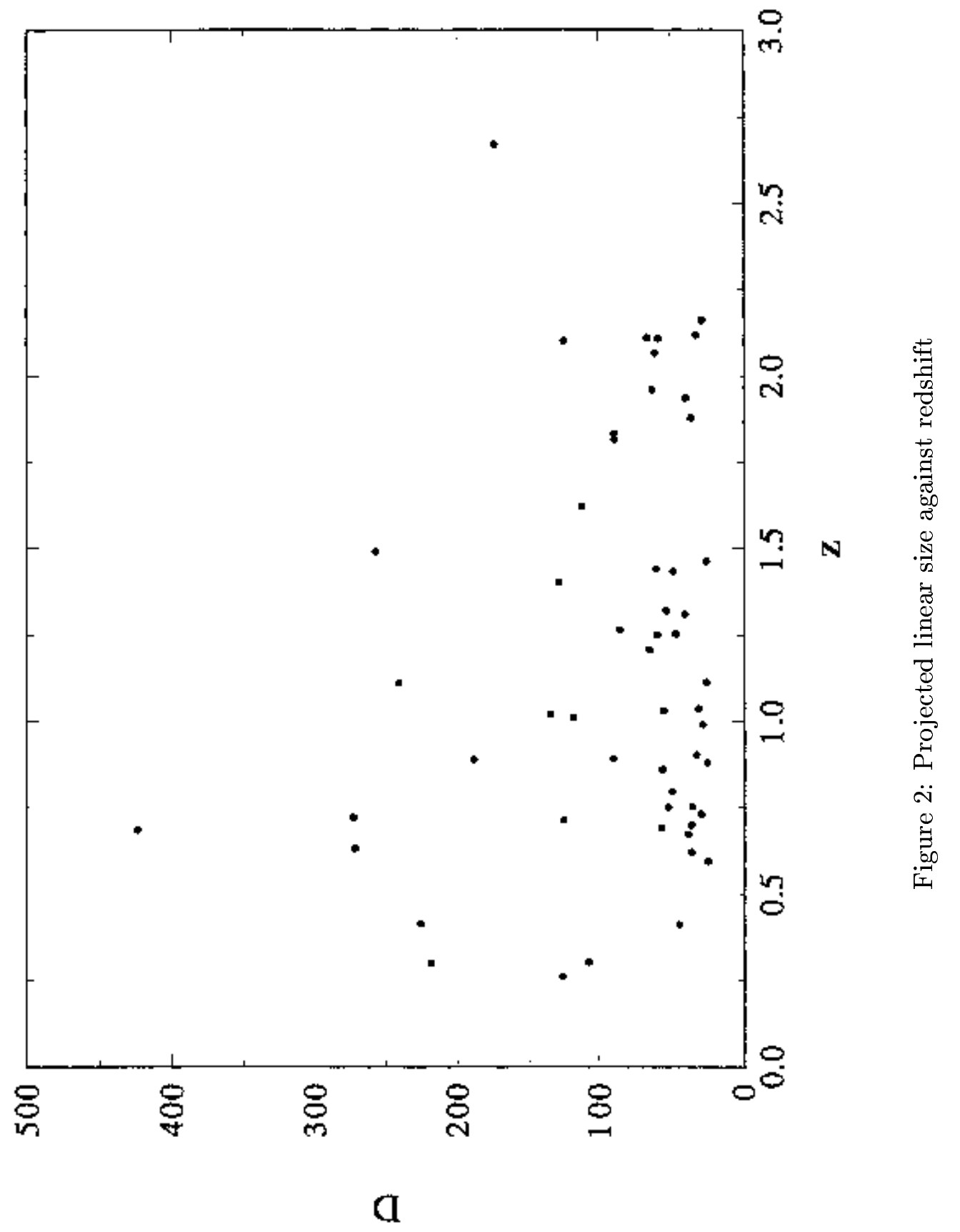




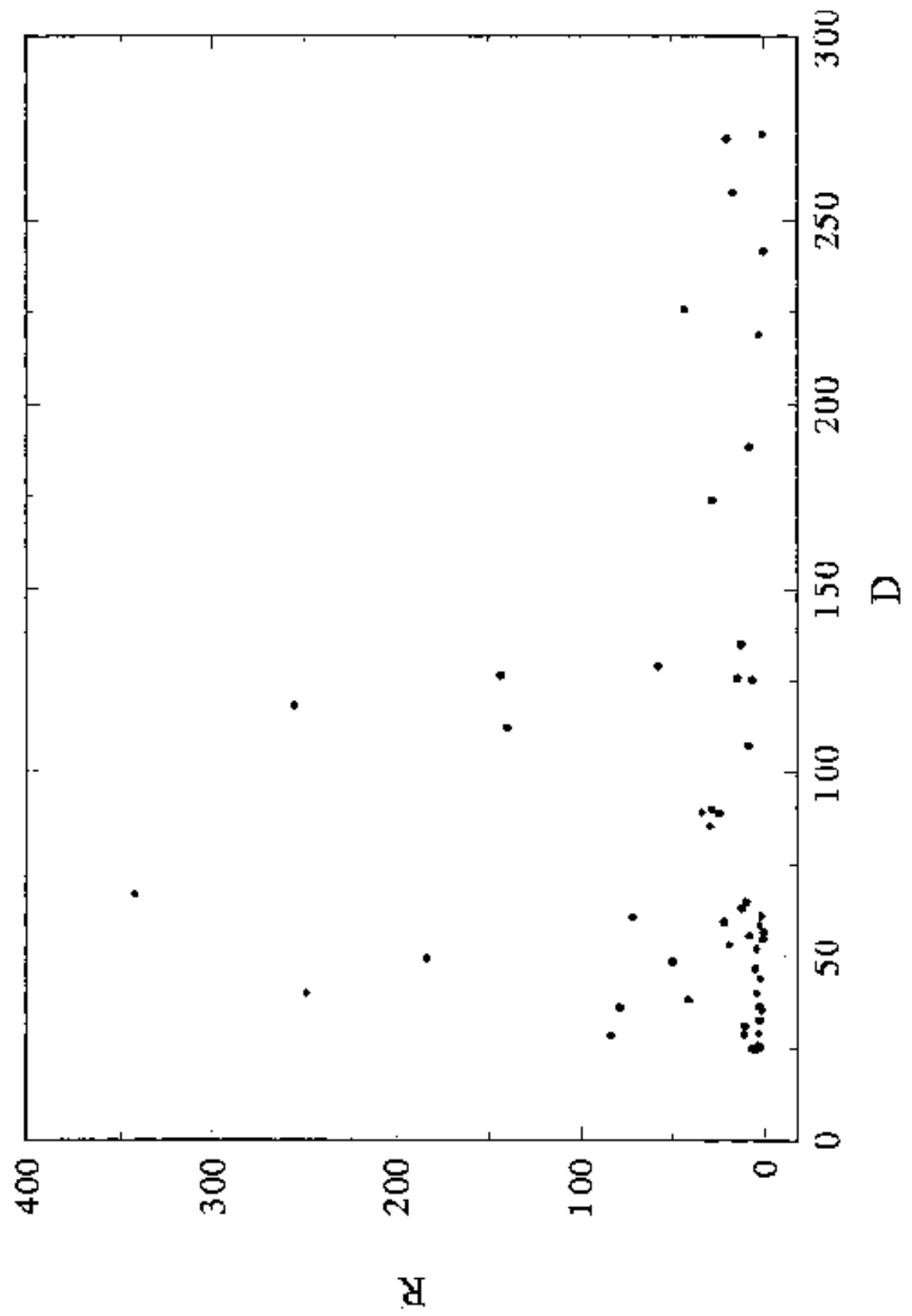

ป̂. 